Pacific Journal of Mathematic 


\title{
A NOTE ON DAVID HARRISON'S THEORY OF PREPRIMES
}

\author{
D. W. Dubois
}

\begin{abstract}
A Stone ring is a partially ordered ring $K$ with unit element 1 satisfying (1) 1 is positive; (2) for every $x$ in $K$ there exists a natural number $n$ such that $n \cdot 1-x$ belongs to $K$; and (3) if $1+n x$ is positive for all natural numbers $n$ then $x$ is positive. Our first theorem: Every Stone ring is order-isomorphic with a subring of the ring of all continuous real functions on some compact Hausdorff space, with the usual partial order. A corollary is a theorem first proved by Harrison: Let $K$ be a partially ordered ring satisfying conditions (1) and (2), and suppose the positive cone of $K$ is maximal in the family of all subsets of $K$ which exclude -1 and are closed under addition and multiplication. Then $K$ is order-isomorphic with a subring of the reals.
\end{abstract}

The present paper is inspired by David Harrison's recently begun program of arithmetical ring theory where the basic objects are primes and preprimes; the positive cones of a ring are example of preprimes. Throughout the paper, $K$ will be a ring with unit element 1 , and $N$ will denote the set of positive integers. A preprime $P$ in $K$ is a nonempty subset of $K$ excluding -1 and closed under addition and multiplication. A prime in $K$ is a preprime maximal relative to set inclusion. A preprime $P$ is infinite provided it contains both zero and 1 , and is conic if $P \cap(-P)=\{0\}$. A conic preprime is simply a positive cone and induces a partial order: $x \geqq y \Leftrightarrow y \leqq x \Leftrightarrow x-y \in P$. A preprime $P$ is Archimedean if for all $x$ in $K$ there exists a natural number $n$ with $n-x$ in $P$, (condition (2) in the definition of Stone ring) and is $(A C)$ if from $1+n x \in P$ for all $n \in N$ follows $x \in P$ (condition (3)). We redefine a Stone ring as a pair $\langle K, P\rangle$ where $P$ is an infinite conic Archimedean $(A C)$ preprime in $K$. An imbedding of $\langle K, P\rangle$ in $\left\langle K^{\prime}, P^{\prime}\right\rangle$ is an injective ring homomorphism $\psi: K \rightarrow K^{\prime}$ such that $P=\psi^{-1}\left(P^{\prime}\right)$. If $X$ is a compact Hausdorff space, $C(X)$ denotes the ring of all continuous real functions on $X, P(X)$ denotes the subset of nonnegative functions. If $K$ is any subring of $C(X)$ then $\langle K, K \cap P(X)\rangle$ is a Stone ring. The principal tool in the proof of Theorem 1 is the Stone-Kadison ordered algebra theorem [3; Theorem 3.1], which characterizes $C(X)$ as a complete Archimedean ordered algebra. To imbed a Stone ring $\langle K, P\rangle$ in such an algebra we show that $K$ is torsionfree, imbed it in a divisible ring $K_{N}$, put a norm on $K_{N}$ and then complete it to $K^{*}$. At each step we have an imbedding of Stone rings: 


$$
\langle K, P\rangle \rightarrow\left\langle K_{N}, P_{N}\right\rangle \rightarrow\left\langle K^{*}, P^{*}\right\rangle \rightarrow\langle C(X), P(X)\rangle,
$$

where the last is Kadison's order-isomorphism. If $P$ is a prime then so is $P_{N}$. [An order-isomorphism is an imbedding onto.]

In the proofs following, $\langle K, P\rangle$ is a Stone ring, $N$ is the set of all positive integers.

\section{Proposition 1. If $n \in N, a \in K$, and $n a \geqq 0$ then $a \geqq 0$.}

Proof. By the unique factorization in $N$, it is enough to prove the proposition for the case where $n$ is a prime number. Suppose for all primes $q<p$ and all $a \in K, q a \geqq 0$ implies $a \geqq 0$. Then for all $n<p$ and all $a \in K, n a \geqq 0$ implies $a \geqq 0$. Now suppose that $p a \geqq 0$ but $a \geq 0$. By the Archimedean property choose $m$ in $N$ with $m+a \geqq 0, x=m-1+\geqq 0$. Then $p x \geqq 0,1+x \geqq 0$ and for all $n$ in $N, 1+(p n+d) x \geqq 0$, if $d=0$ or $d=1$. [In case $p=2$ this implies that $1+k x \geqq 0$ for all $k$, so $x \geqq 0$ by $(A C)$, a contradiction; hence $2 a \geqq 0$ implies $a \geqq 0$.] Now let $1<d<p$, with $d$ in $N$. Since $p$ is a prime there exists $e$ in $N$, with $1<e<p, e d=1+p n$, for some $n$ in $N$. Then $e(1+d x)=e+(1+p n) x=(e-1)+(1+x)+$ $(p n x) \geqq 0$. Since $e<p$ this implies that $1+d x \geqq 0$. So for all $k$ in $N, 1+(p k+d) x=1+d x+p k x \geqq 0,0 \leqq d \leqq p-1$. That is, $1+$ $n x \geqq 0$ for all $n$ in $N$. By $(A C)$ again, $x \geqq 0$, a contradiction. So $a \geqq 0$ and the induction is complete.

Now put

$$
\begin{aligned}
& K_{N}=Q \otimes K=\{k / n ; k \in K, n \in N\}, \\
& P_{N}=\{p / n ; p \in P, n \in N\} . \\
& \varphi: K \rightarrow K_{N}, \varphi(k)=k / 1 .
\end{aligned}
$$

Proposition 2. $\left\langle K_{N}, P_{N}\right\rangle$ is also a Stone ring. If $P$ is a prime then so is $P_{N}$. $\varphi$ is an imbedding.

Proof. That $\varphi$ is injective follows from Proposition 1 . If $k / n$, for $k$ in $K, n$ in $N$, belongs to $P_{N}$, then $k$ belongs to $P$. For $k / n$ in $P_{N}$ implies $k / n=p / m$, for some $p$ in $P, m$ in $N$, so $m k=n p \in P$. By Proposition $1, k \in P$. Hence $\varphi$ is an imbedding. The preprime, infinite, and conical properties of $P_{N}$ follow easily from the corresponding properties for $P$. For the Archimedean property, let $k / m$ be arbitrary in $K_{N}$ ( $k$ in $K, m$ in $N$ ) and choose $n$ in $N$ with $n>k$. Then $n-k / m=(n m-k) / m$ belongs to $P_{N}$ since $n m>k, m \in N$. Now if $1+n(k / m) \geqq 0$ holds in $K_{N}$, with $m$ in $N, k$ in $K$, and for all $n$ in $N$, then for all $n, \varphi(1+n k)=1+m n(k / m) \in P_{N}$. Since $\varphi$ is an imbedding, $1+n k \in P$. By the $(A C)$ property for $P, k \in P, k / m \in P_{N}$. 
This establishes $(A C)$ for $P_{N}$. Finally let $P^{\prime}$ be a preprime containing $P_{N}$ and let $P_{1}=\varphi^{-1}\left(P^{\prime}\right)$. Then $P_{1}$ is a preprime containing $P$. If the first containment is proper so is the second. This proves that if $P$ is a prime then $P_{N}$ is a prime.

Note. The additive group of $K_{N}$ is divisible. If $K$ were already divisible then $\varphi$ would be an order-isomorphism of $\langle K, P\rangle$ onto $\left\langle K_{N}, P_{N}\right\rangle$. The rational multiples of 1 in $K_{N}$ form a field orderisomorphic with $Q$.

Now define $t$ on $K_{N}$ by

$$
t(x)=\inf \{r ;-r<x<r, r \in Q\} .
$$

Proposition 3. The function $t$ is a norm on $K_{N}$ :

(a) $t(x) \geqq 0 ; t(x)=0$ if and only if $x=0$.

(b) $t(x+y) \leqq t(x)+t(y)$.

(c) $t(x y) \leqq t(x) t(y)$

(d) $t(r x)=|r| t(x)$ for $r$ in $Q$.

Put $K^{*}$ equal to the completion of $K_{N}, P^{*}$ equal to the closure of $P_{N}$ in $K^{*}$. Then $\left\langle K^{*}, P^{*}\right\rangle$ is a Stone ring and an Archimedean ordered algebra as defined by Kadison.

Proof. The property (a) follows from $(A C)$. Properties (b) and (c) follow from: if $-r<x<r,-s<y<s$ then $-(r+s)<x+$ $y<r+s$, and $-r s<x y<r s$. See [1], $\S 2$. The proofs there make no use of commutativity or of multiplicative inverses. Property (d) is a consequence of: $-r<x<r$ if and only if $-r q<q x<r q$, where $q$ is a positive rational. It is clear that $t(-x)=t(x)$ and for rational $r, t(r)=|r|$. We now identify $K_{N}$ with its injection in its completion $K^{*}$ and note that $P^{*} \cap K_{N}=P_{N}$ : for if $k \in P^{*} \cap K_{N}$ then $k=\lim p_{n}$, $p_{n} \in P_{N}$, and $p_{n}$ may be chosen so that $-1 / n<k-p_{n}<1 / n$ for all $n \in N$; it follows that $1+n k>n p_{n}>0$ for all $n \in N$ and thence by $(A C)$ that $k \in P_{N}$. The reverse inclusion is obvious. It remains to prove that $P^{*}$ is an infinite conical Archimedean $(A C)$ preprime. It is certainly closed under addition and multiplication. Let $x \in P^{*} \cap\left(-P^{*}\right)$. Then there exist positive sequences $p_{n}$ and $q_{n}$ with $x=\lim p_{n},-x=$ $\lim q_{n}, 0=\lim \left(p_{n}+q_{n}\right)$. Thus if $\varepsilon$ is any positive real then for all large $n, 0 \leqq p_{n} \leqq p_{n}+q_{n}<\varepsilon$, so $x=\lim p_{n}=0 ; P^{*}$ is therefore conical. Let $x_{n} \in K_{N}$, with $x=\lim x_{n}$. The Cauchy sequence $\left\{x_{n}\right\}$ is bounded in norm so there exists an integer $m$ with $m>x_{n}$ for all $n$. Hence $m-x=\lim \left(m-x_{n}\right) \in P^{*}, m>x$. This shows $P^{*}$ is Archimedean. Now let $1+n x \in P^{*}$ for all $n$ in $N\left(x \in K^{*}\right)$. $P^{*}$, as closure of $P$, is closed and hence contains $x=\lim (x+1 / n)$, since $x+1 / n$ belongs to $P^{*}$. Thus $P^{*}$ is $(A C)$. That $1 \in P^{*}$ and $-1 \notin P^{*}$ are obvious, and 
it has now been proved that $\left\langle K^{*}, P^{*}\right\rangle$ is a Stone ring. The closure of $Q$ in $K^{*}$ is (order-isomorphic with) the reals $R$. Using $t$ for the induced norm in $K^{*}$ we have

(e) $t(r x)=|\boldsymbol{r}| t(x)$ for all $r$ in $R$.

$R$ is contained in the center of $K^{*}$ and so $\left\langle K^{*}, P^{*}\right\rangle$ is an algebra over the reals. For the sake of completeness we list Kadison's axioms for an Archimedean ordered algebra. Each is obviously satisfied by $\left\langle K^{*}, P^{*}\right\rangle$ with $e=1$.

1. $K^{*}$ is a real algebra with unit $e$.

2. $P^{*}$ is closed under addition, multiplication, and multiplication by positive reals.

3. For every $x$ in $K^{*}$ there exists a positive real $r$ with $r e>x$.

4. If $r e \geqq x$ for all positive real $r$, then $x \leqq 0$.

An Archimedean ordered algebra is complete if and only if it is complete in our norm $t$. Thus $\left\langle K^{*}, P^{*}\right\rangle$ is a complete Archimedean ordered algebra. Collecting results of Propositions 1, 2, and 3 and applying Theorem 3.1 of Kadison we get our Theorem 1 .

Now we are ready to prove the corollary. As we remarked earlier, Harrison showed that a prime $P$ satisfying the hypotheses there is also $(A C)$. By Proposition 2, $P_{N}$ is also a prime. Now identify each of $\langle K, P\rangle,\left\langle K_{N}, P_{N}\right\rangle,\left\langle K^{*}, P^{*}\right\rangle$ with its imbedding in $\langle C(X), P(X)\rangle$, so that $P\left(P_{N}\right)$ is the set of all nonnegative functions in $K\left(K_{N}\right)$. The proof is completed by showing that $X$ is a singleton. Suppose that $x$ and $y$ are distinct points of $X$. Since $X$ is normal and $K_{N}$ is dense in $C(X)$, Urysohn's lemma guarantees that there is a function $f$ in $K_{N}$ with $f(x)>0, f(y)<0$. Then $P^{\prime}=\left\{g ; g \in K_{N}\right.$ and $\left.g(x) \geqq 0\right\}$ is a preprime in $K_{N}$ containing $P_{N}$ and $f$, while $f$ is not in $P_{N}$. This contradicts the primality of $P_{N}$ and the corollary is proved.

Two Examples. 1. Example of a ring $\langle K, P\rangle$ where all the conditions of Theorem 1 hold for $P$ except the Archimedean condition. Let $K$ be the ring of all $2 \times 2$ real matrices, $P$ the set of matrices with every entry nonnegative.

2. Example of a ring $\left\langle K^{\prime}, P^{\prime}\right\rangle$ where $P^{\prime}$ satisfies all except the condition $(A C)$. Put $K^{\prime}$ equal to the set of all triangular $2 \times 2$ matrices over $R$ and let $P^{\prime}$ be the subset consisting of 0 and all matrices with strictly positive diagonal entries. Thus if either of the Archimedean conditions is omitted then commutativity cannot be deduced. 


\section{REFERENCES}

1. D. W. Dubois, On partly ordered fields, Proc. Amer. Math. Soc. 7 (1956), 918-930.

2. D. K. Harrison, Finite and infinite primes for rings and fields. (Mimeographed preprint)

3. R. V. Kadison, A representation theory for commutative topological algebra, Memoirs of Amer. Math. Soc. No. 7 (1951).

Received April 28, 1966. 



\section{PACIFIC JOURNAL OF MATHEMATICS}

\section{EDITORS}

H. SAMELSON

Stanford University

Stanford, California

J. P. JANS

University of Washington

Seattle, Washington 98105
J. DugundJI

University of Southern California

Los Angeles, California 90007

RICHARD ARENS

University of California

Los Angeles, California 90024

\section{ASSOCIATE EDITORS}

E. F. BECKENBACH

B. H. NeumanN

F WolF

K. YOSIDA

\section{SUPPORTING INSTITUTIONS}

UNIVERSITY OF BRITISH COLUMBIA

CALIFORNIA INSTITUTE OF TECHNOLOGY

UNIVERSITY OF CALIFORNIA

MONTANA STATE UNIVERSITY

UNIVERSITY OF NEVADA

NEW MEXICO STATE UNIVERSITY

OREGON STATE UNIVERSITY

UNIVERSITY OF OREGON

OSAKA UNIVERSITY

UNIVERSITY OF SOUTHERN CALIFORNIA
STANFORD UNIVERSITY

UNIVERSITY OF TOKYO

UNIVERSITY OF UTAH

WASHINGTON STATE UNIVERSITY

UNIVERSITY OF WASHINGTON

AMERICAN MATHEMATICAL SOCIETY CHEVRON RESEARCH CORPORATION TRW SYSTEMS

NAVAL ORDNANCE TEST STATION

Printed in Japan by International Academic Printing Co., Ltd., Tokyo Japan 


\section{Pacific Journal of Mathematics}

\section{Vol. 21, No. $1 \quad$ November, 1967}

Friedrich-Wilhelm Bauer, Der Hurewicz-Satz................... 1

D. W. Dubois, A note on David Harrison's theory of preprimes . ......... 15

Bert E. Fristedt, Sample function behavior of increasing processes with stationary, independent increments ..................... 21

Minoru Hasegawa, On the convergence of resolvents of operators....... 35

Søren Glud Johansen, The descriptive approach to the derivative of a set function with respect to a $\sigma$-lattice ....................... 49

John Frank Charles Kingman, Completely random measures ............ 59

Tilla Weinstein, Surfaces harmonically immersed in $E^{3} \ldots \ldots \ldots \ldots \ldots . . \ldots 9$

Hikosaburo Komatsu, Fractional powers of operators. II. Interpolation spaces ......................................... 89

Edward Milton Landesman, Hilbert-space methods in elliptic partial differential equations ...................................... 113

O. Carruth McGehee, Certain isomorphisms between quotients of a group algebra ........................................ 133

DeWayne Stanley Nymann, Dedekind groups .................. 153

Sidney Charles Port, Hitting times for transient stable processes ......... 161

Ralph Tyrrell Rockafellar, Duality and stability in extremum problems involving convex functions . ............................ 167

Philip C. Tonne, Power-series and Hausdorff matrices . . .............. 189 